\title{
EXTRACTION OF ROCKY DESERTIFICATION FROM DISP IMAGERY: A CASE STUDY OF LIUPANSHUI, GUIZHOU, CHINA
}

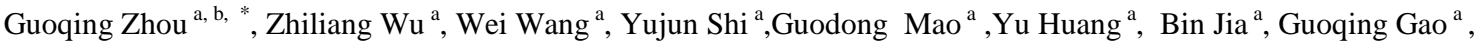 \\ Pengyun Chen ${ }^{\mathrm{a}}$ \\ ${ }^{a}$ Guangxi Key Laboratory for Spatial Information and Geomatics, Guilin University of Technology, \\ Guilin, Guangxi, 541004, China; glitezhou@ yahoo.com \\ ${ }^{\mathrm{b}}$ College of Precision Instrument and Opto-Electronic Engineering, Tianjin University, Tianjin, 300072, \\ China; zrt65@tju.edu.cn
}

\section{Commission III, WG III/6}

KEY WORDS: DISP image, Geometric rectification, Image mosaic, Karst ,Rocky desertification

\begin{abstract}
:
Karst rocky desertification is a typical type of land degradation in Guizhou Province, China. It causes great ecological and economical implications to the local people. This paper utilized the declassified intelligence satellite photography (DISP) of 1960s to extract the karst rocky desertification area to analyze the early situation of karst rocky desertification in Liupanshui, Guizhou, China. Due to the lack of ground control points and parameters of the satellite, a polynomial orthographic correction model with considering altitude difference correction is proposed for orthorectification of DISP imagery. With the proposed model, the 96 DISP images from four missions are orthorectified. The images are assembled into a seamless image map of the karst area of Guizhou, China. The assembled image map is produced to thematic map of karst rocky desertification by visual interpretation in Liupanshui city. With the assembled image map, extraction of rocky desertification is conducted.
\end{abstract}

\section{INTRODUCTION}

Karst rocky desertification has become an important topic to prevent soil erosion, restore vegetation, and pull farmers out of poverty in the karst areas of Southwestern China. Guizhou Province has the largest desertification area, suffering the most homogeneous, the deepest and the most serious from rocky desertification (Zhao, 2011). The rocky desertification area in Guizhou covers $37597.36 \mathrm{~km}^{2}$, accounting for $21.34 \%$ of the entire land area of Guizhou Province, approximately $34.47 \%$ of the total area of karst of China. (http://www.gzrd.gov.cn/gzdt /gdgz/2207.shtml). Therefore, the use of DISP image to draw a typical karst terrain of Guizhou rocky desertification distribution map is of great significance for scientific research on the history of rocky desertification change and development characteristics.

\section{DATA}

\subsection{Study Area}

Liupanshui is located in the west of Guizhou Province, in the east longitude $104^{\circ} 18^{\prime} 20^{\prime \prime} \sim 105^{\circ} 42^{\prime} 50 "$, latitude $25^{\circ} 19^{\prime} 44$ " $26^{\circ} 55^{\prime} 33^{\prime \prime}$ ( Figure 1). Its total area is $9,926 \mathrm{~km}^{2}$, accounting for the province's total area of $5.63 \%$. Liupanshui has the most severe karst rocky desertification in Guizhou, the karst area of which was $63.18 \%$ of total city area until 2005. The area of karst rocky desertification is $3,460 \mathrm{~km}^{2}$, representing $34.8 \%$ of the total area of the city. Among them, the area of very severe rocky desertification is $565 \mathrm{~km}^{2}$. It is a typical karst ecosystem with a wide range of distribution in karst, complete morphological types and obvious regional distribution.

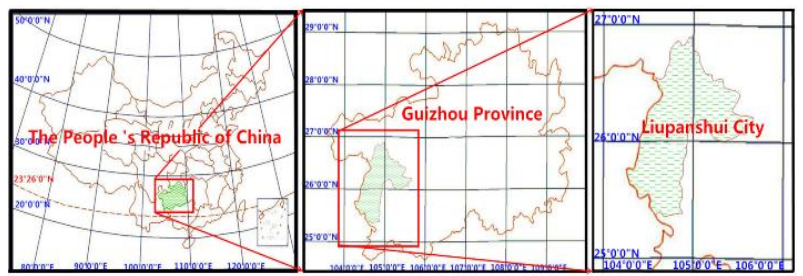

Figure 1. The location of Liupanshui

\subsection{DISP Image}

Declassified intelligence satellite photographs (DISP) is the first generation of photographic reconnaissance satellites between 1960 and 1972(Zhou, 2016). The systems include CORONA, ARGON and LANYARD. In this paper the CORONA imagery was used. Early DISP images were served in both military and intelligence. After President Bill Clinton signed an executive order of intelligence declassified, it became a public satellite image of decrypting information. More than 990,000 images collected from 1959 to 1980 were declassified by this executive order. Only NARA and the US Geological Survey (USGS) were authorized to provide access and product support for the February 1995 and September 2002 datasets(McDonald, 1995). The DISPs used in this paper are all from the US Geological Survey Bureau website.

This paper uses the declassified intelligence satellite photographs image (DISP) from the US Geological Survey Website as the basic image to study the karst region of Guizhou Province in the 1960s. The 96 images come from five different Missions of CORONA satellite, including 1035-1 Mission, 1102-2 Mission, 1106-1 Mission and 1114-2 Mission (Figure

\footnotetext{
${ }^{*}$ Corresponding author: Guoqing Zhou; Tel. /Fax: +86(773)5896073;

E-mail: glitezhou@yahoo.com
} 
2).The image DS1106-2119DF071-097 accessed by Mission1106-2 fully cover the research area of Liupanshui City.

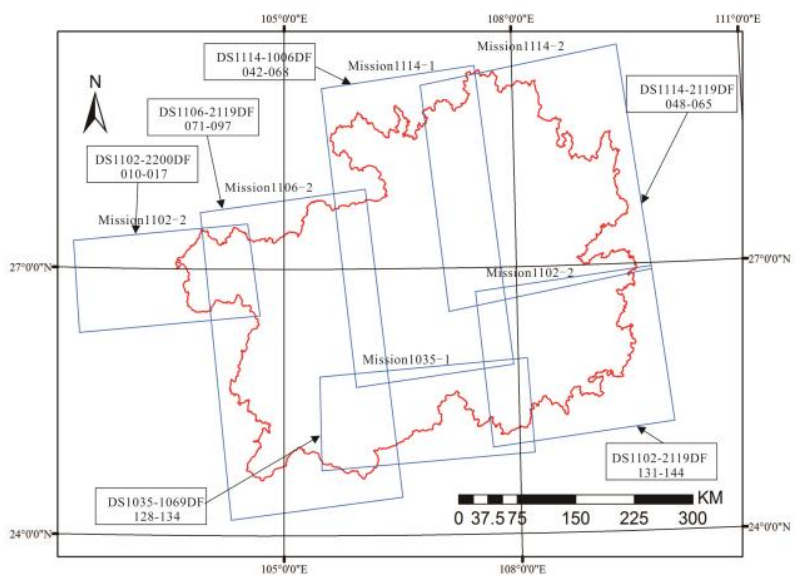

Figure 2. The coverage area of DISP imagery in Guizhou

\section{DISP IMAGE DATA PROCESSING}

\subsection{Geometric Rectification of the Images}

The geometric distortions of remote sensing images are systematic and non-systematic, mainly including satellite image acquisition methods, the sensor orientation elements, topography and atmospheric refraction (Yang, 2002). Geometric distortion of the remote sensing image changes the geometric location, size and shape of some objects, and therefore cannot be directly used in specific research applications. Therefore, remote sensing images must be geometrically corrected prior to specific application studies. Geometric correction is to correct the geometric distortion of the image, which can be divided into two types: geometric approximate correction and geometric accurate correction. Geometric approximate correction is to correct systematic errors. Geometric accurate correction is a geometric correction by adding control points, which uses a mathematical model to simulate geometric distortion of remote sensing images and then apply this model to perform geometric correction through the corresponding geometric distortion models that are obtained by distorting the corresponding points between the remote sensing image and the standard map (Amini, 2005).

Due to the parameters of the satellite are unknown, such as the interior and external orientation parameters and the camera lens distortion parameters, the correction method that requires satellite parameters cannot be used when correcting the DISP image. The polynomial model does not depend on the parameters of the satellite, only a sufficient amount of control points, so the model is suitable for dealing with these historical images. As the study area belongs to the karst topography, multi-hilly, peak cluster depression and other terrain, the elevation in the image of the distortions will be an important element. In this paper, the polynomial model is added to the elevation, and a 3-dimensional 2-order polynomial model is established to correct the deformation caused by the height difference. The 3-dimensional polynomial expression is as follows:

$$
\left\{\begin{array}{l}
X_{I}=\sum_{i=0}^{M} \sum_{j=0}^{N} \sum_{k=0}^{Q} a_{i j} \cdot X_{G}^{i} Y_{G}^{j} Z_{G}^{k} \\
Y_{I}=\sum_{i=0}^{M} \sum_{j=0}^{N} \sum_{k=0}^{Q} b_{i j} \cdot X_{G}^{i} Y_{G}^{j} Z_{G}^{k}
\end{array}\right.
$$

Then, Equation (1)can be rewritten as follows:

$$
\left\{\begin{array}{l}
X_{I}=a_{0}+a_{1} X_{G}+a_{2} Y_{G}+a_{3} Z_{G}+a_{4} X_{G}^{2}+ \\
a_{5} Y_{G}^{2}+a_{6} Z_{G}^{2}+a_{7} X_{G} Y_{G}+a_{8} X_{G} Z_{G}+a_{9} Y_{G} Z_{G}+\ldots \\
Y_{I}=b_{0}+b_{1} X_{G}+b_{2} Y_{G}+b_{3} Z_{G}+b_{4} X_{G}^{2}+ \\
b_{5} Y_{G}^{2}+b_{6} Z_{G}^{2}+b_{7} X_{G} Y_{G}+b_{8} X_{G} Z_{G}+b_{9} Y_{G} Z_{G}+\ldots
\end{array}\right.
$$

Where, $\left(\mathrm{X}_{\mathrm{I}}, \mathrm{Y}_{\mathrm{I}}\right)$ represents image point coordinates of the imagery; $\left(\mathrm{X}_{\mathrm{G}}, \mathrm{Y}_{\mathrm{G}}, \mathrm{Z}_{\mathrm{G}}\right)$ represents the ground point coordinates. The relationship between $\mathrm{N}$ and $\mathrm{n}$ can be expressed as:

$$
N=\frac{(n+1)+(n+2)}{2}
$$

Where, $\mathrm{N}$ represents the number of coefficient, $\mathrm{n}$ represents the degree of polynomial

\subsection{Selection of Control Points}

The selection of ground control points is the most essential step in geometric correction of remote sensing imagery. As historical images have been more than half a century ago, it difficult to obtain the contemporary high-precision map of the image. This paper selects the Google Earth image as the reference image for the control points. Finding the current corresponding feature point on historical imagery map not only needs manual identification, but also needs historical events to identify the stable surface features. In order to select accurate feature points, try to select those with clear indicators, such as bridges, building corners, intersections and other road inflection points. The control points should be distributed on the image as evenly as possible, so as not to overly centralize control points, as missing or too few control points in some place would cause big error and deformation.

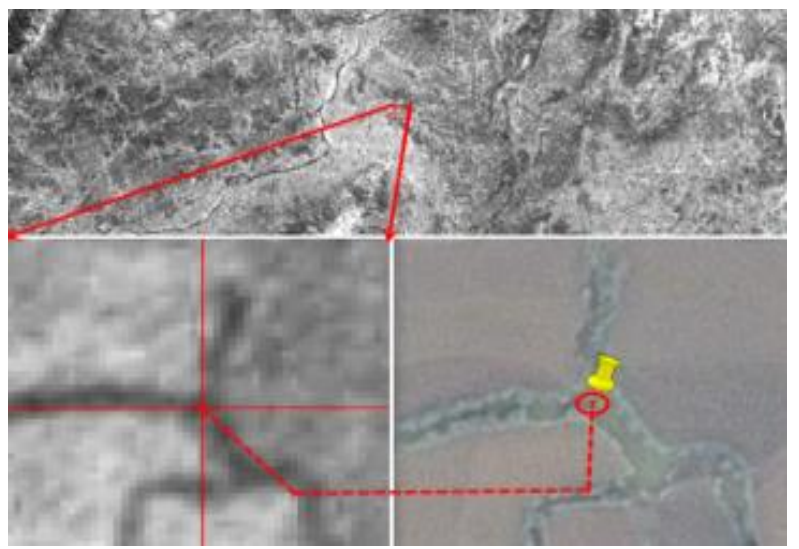

Figure 3. Selection of control points on Google Earth

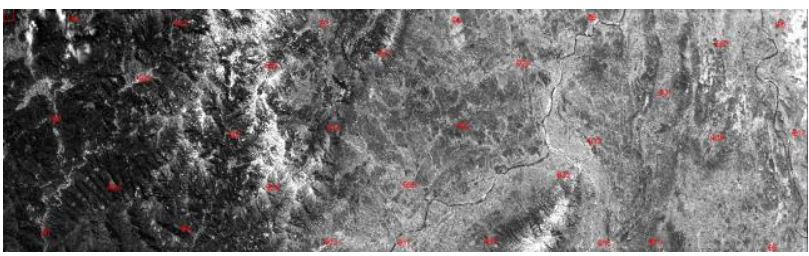

Figure 4. Distribution of selected control points 


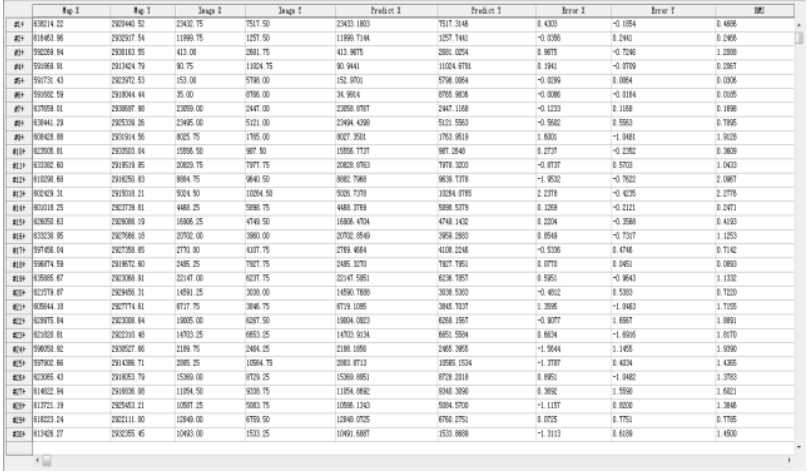

Figure 5. RMS of selected control points

\subsection{Image Mosaicking}

In the application research of remote sensing image, the research scope is beyond the coverage of single scene image. In order to satisfy needs of application research, better processing, interpretation and analysis of remote sensing image information, it is often necessary to have some overlap of the adjacent two or more remote sensing images into a larger range of images (Wen, 2009).

As with mosaic produced from satellite data, there are two fundamental problems: geometric, A method based on overlapped image is used to solve the problem in this paper, smoothing the transition of image overlay and realizing the seamless joint of remote sensing image.

Each scene image is divided into a, b, c and d frames when the USGS provides data. When mosaicking, the overlapping area of four images is used to mosaic. The mosaicing process is shown in Figure 6,7,8:

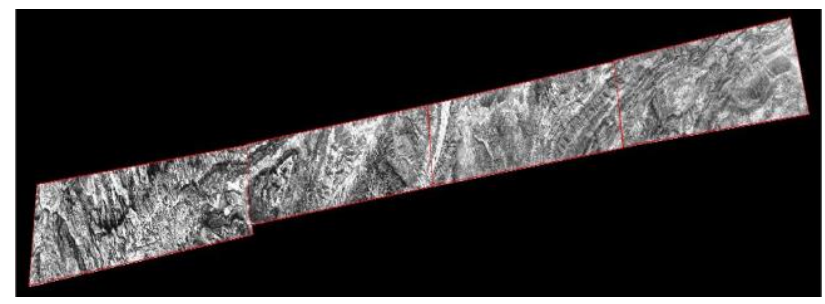

Figure 6 . The selection of image seamline

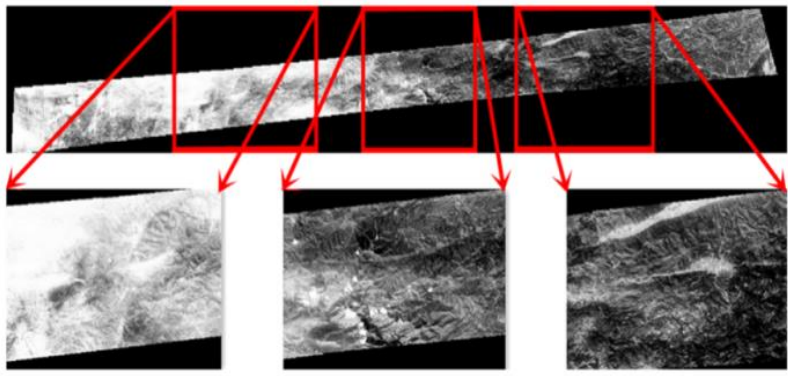

Figure 7 . Single scene imagery mosaic effect

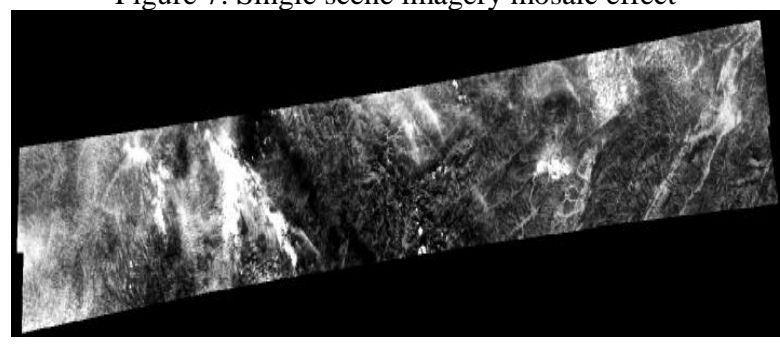

Figure 8. Double scene imagery mosaic effect
Due to the CORONA series of images used in this paper come from a different time, seamline and intensity cannot be eliminated. To eliminate the difference, this paper use a histogram equalization method to radiometric balancing (Zhou, 2016). The radiometric balancing process is shown in Figure 9,10:

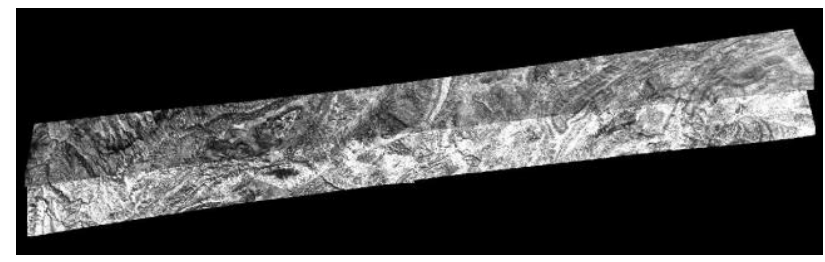

Figure 9. Image mosaic without radiometric balancing.

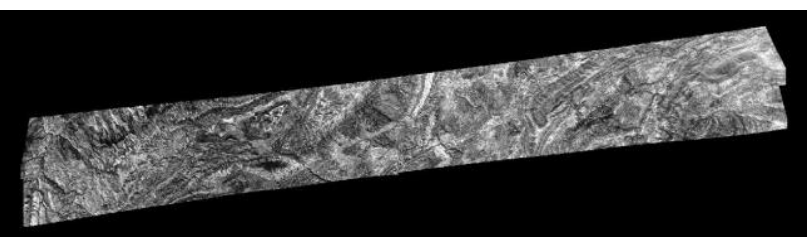

Figure 10. Image mosaic after radiometric balancing.

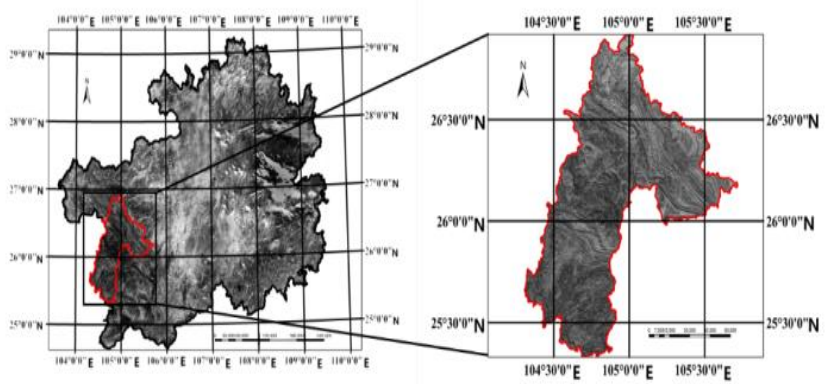

Fig.11 High Resolution Panchromatic Image of Liupanshui in $1960 \mathrm{~s}$

\section{EXPERIMENT RESULT AND ANALYSIS}

\subsection{The Establishment of Visual Interpretation Signs}

In some cases, manual interpretation and other information should be used together to further define. When the computer automatically identifies some of the spots, some abundant graphics spots will be monitored, and then there is a need for visual interpretation to remove those redundant. In the automatic recognition, large computer graphics will not be omitted, but some small spots may be missing, then it is necessary to add a visual interpretation (Wang, 2003). Due to the effects of many factors, such as the full color of DISP image, relatively less information of Pope, long history, the object reference lack of the corresponding object and so on, it cannot achieve the ideal result simply by using computer. In this paper, the extraction of karst rocky desertification in the 1960s in Liupanshui is based on ENVI, ERDAS and ARCGIS, besides the method of human-computer interaction interpretation is adopted.

The interpretation sign of remote sensing image refers to the various features of remote sensing image which can reflect and represent the feature information and can help the interpreter identify the target object on the remote sensing image. There are two types of interpretation signs: a direct interpretation signs and indirect interpretation signs. Direct interpretation signs usually are the target size, shape, shadow, tone, texture, graphics and location and the surrounding relationship; indirect 
interpretation signs are those that can indirectly reflect the target features of remote sensing images(Jiang, 2002). In order to establish the interpretation of the signs, our team members conducted a field survey to obtain the real image of rocky desertification area. A visual interpretation of signs is established according to the corresponding position (Figure 12 and table 1).

\begin{tabular}{|c|c|c|c|}
\hline $\begin{array}{c}\text { The of } \\
\text { rocky } \\
\text { desertificati } \\
\text {--on }\end{array}$ & $\begin{array}{c}\text { Slope } \\
\left({ }^{0}\right)\end{array}$ & $\begin{array}{c}\text { Sign of visual } \\
\text { interpretation }\end{array}$ & $\begin{array}{c}\text { The lower } \\
\text { part of } \\
\text { hillside and } \\
\text { the shape is } \\
\text { irregular } \\
\text { severe }\end{array}$ \\
\hline moderately \\
severe
\end{tabular}

Table 1.The Establishment of Visual Interpretation Signs
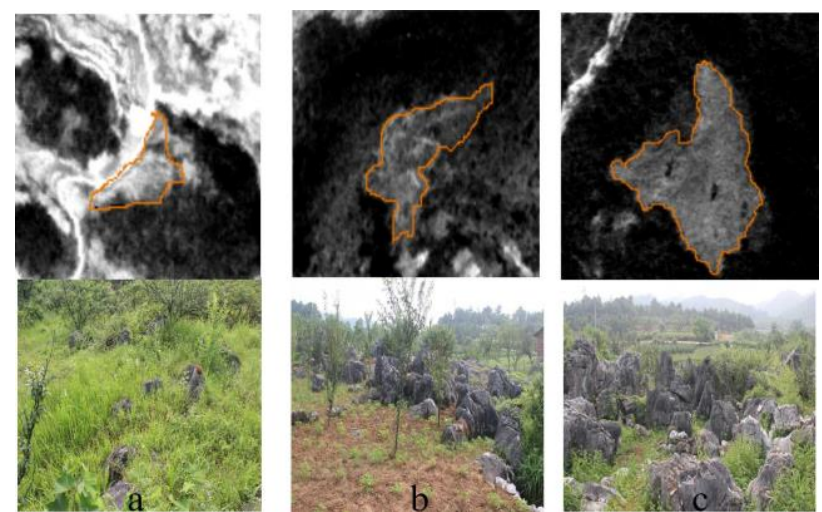

Figure 12. Real images of rocky desertification area

(a. mildly severe, b. moderately severe, c. severe)

\subsection{Extraction of Karst Rocky Desertification}

This article interprets the karst rocky desertification classification system visually by man-machine interactive interpretation. In order to improve the accuracy, some data will be used directly, including 2005 rocky desertification thematic map, slope thematic map and DISP images, in the process of depicting rocky desertification area.

In terms of the relative position, the rocky desertification area in modern times contains the rocky desertification area in the 1960 s, so it is possible to apply the thematic map of rocky desertification to determine the area of rocky desertification in the 1960s. In addition, from the interpretation of the information, we can see that the 1960s rocky desertification area will be less than $22^{\circ}$. Therefore, according to the overlapping area of modern rocky desertification area and $22^{\circ}$ slope map, it is possible to reduce the range of interpretation and improve the accuracy of interpretation by obtaining the rocky desertification area in the 1960s. Finally, according to the interpretation of signs, we finally depict the rocky desertification area. Images of karst areas in Liupanshui city in 1960 s, were cut into several parts according to the vectors of each county. In the 1960s, the area of rocky desertification in Liupanshui reached $4,938.185 \mathrm{~km}^{2}(49.75 \%$ of total city area), with very severe rocky desertification area of $608.50 \mathrm{~km}^{2}$ (6.08\% of total city area); moderate rocky desertification area of $730.55 \mathrm{~km}^{2}$ (7.36\% of total city area); mild rock desertification area of $3,604.13 \mathrm{~km}^{2}$ (36.31\% of total city area). In 2005 , the area of rock desertification in Liupanshui was more than 3,460 $\mathrm{km}^{2}$, which was 1.4 times of that in the 1960s.

Then, the rocky desertification areas were mapped in each county, and the distribution map of rocky desertification in 1960s in Liupanshui City of Guizhou Province was produced (Figure 13,14), while the area of rocky desertification is obtained.

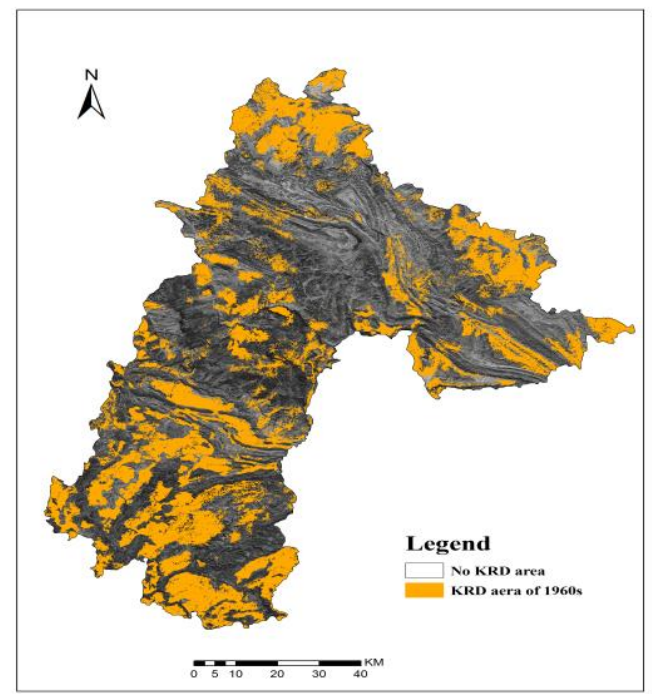

Figure 12.Rocky desertification area of Liupanshui in 1960s

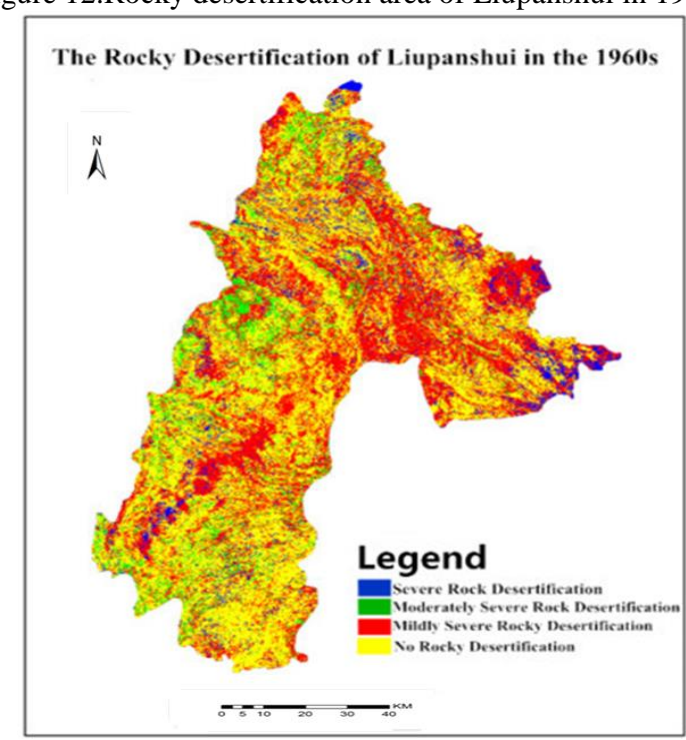

Figure 13. The thematic map of rocky desertification distribution in Liupanshui City in the 1960s

(Yellow: no rocky desertification, Red: mildly severe, Green: moderately severe, Blue: severe) 


\subsection{Accuracy Evaluation of Final Products}

Based on the high resolution images of Google Earth as present reference images and DISP images as historical reference images, we verified the regional classification results of karst rocky desertification in the 1960s (Figure 13). After a lot of image matching tests and on-site inspection of regional map provided by Provincial Land and Environmental Protection Departments of Guizhou province, the precision reached the requirements.
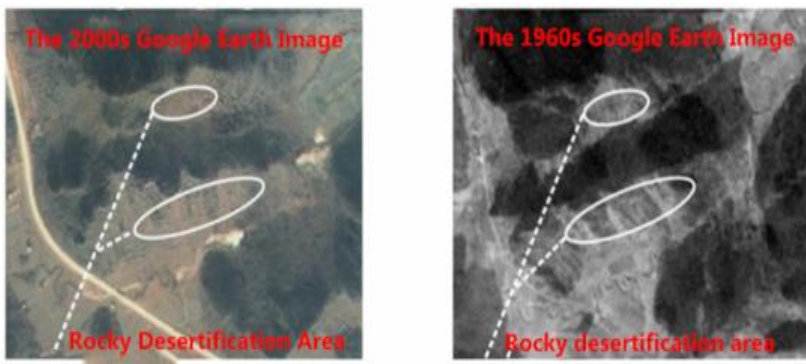

Figure 13. Verification of rocky desertification area extraction

\section{CONCLUSION}

Based on the DISP image processing, the high resolution image mosaic of Guizhou Province in 1960s was completed, and the rocky desertification in the 1960s in Lupanshui area of Guizhou Province was extracted. The experiments have achieved good results and can provide an effective means. for the extraction of ground objects information and the desertification extraction of larger areas in the future.

\section{ACKNOWLEDGEMENT}

This paper is financially supported by the National Natural Science of China under Grant numbers 41431179 and 41162011, The National Key Research and Development Program of China under Grant numbers 2016YFB0502501 and The State Oceanic Administration under Grant numbers [2014]\#58, GuangXi Natural Science Foundation under grant numbers 2015GXNSFDA139032, and 2012GXNSFCB05300; Guangxi Science \& Technology Development Program under the Contract number GuiKeHe 14123001-4, and GuangXi Key Laboratory of Spatial Information and Geomatics Program (Contract No. GuiKeNeng 151400734, 110310801, 120711501, and 130511401), the "BaGuiScholars" program of the provincial government of Guangxi.

\section{REFERENCES}

Shuyan Zhao, 2011.The establishment of legal mechanism for preventing and controlling desertification in Southwest China, Shangqing, pp.215-216.

http://www.gzrd.gov.cn/gzdt/gdgz/2207.shtml

Guoqing Zhou, Tao Yue, Yujun Shi, Rongting Zhang and Jingjin Huang, 2016. Second-Order Polynomial EquationBased BlockAdjustment for Orthorectification of DISP Imagery, Remote Sensing, Remote Sens,pp. 1-18.

McDonald, R. A., 1995. Corona: success for space reconnaissance, a look into the cold war, and arevolution for intelligence. Photogrammetric Engineering \& Remote Sensing, 61(6),pp. $689-720$.
Gaopan Yang, Shihong Guo and Leiping Ren, 2002. Research on geometric correction of high - resolution satellite image. Journal of Computer Knowledge and Technology.

Jiabing Sun, 2003. Principles and application of remote sensing.Wuhan University Publication, Wuhan.

Mengqian Li, 2012. Geometric rectification and accuracy analysis based on worldview-2 and rapid eye data. China University of Geosciences, Beijing.

V. Kratky, 1971. Precision process of ERTS Imagery. Proceedings of ASP-ACSM Fall Convention, San Fransico, Ca, September 7-11, (Falls Church, Va: ASP),pp. 481-514,

V. Kratky, 1989. On-1ine aspects of stereophotogrammetric processing of SPOT images. Photogrammetric Engineering and Remote Sensing, 55,pp. 311-316.

Fraser S.C.,Dare P.M.,Yamaka A, 2004. Digital surface modelling from SPOT5 HRS imagery using the affine projective model. The International Society for Photogrammetry and Remote Sensing, pp.385-388 .

Amini J, \& Hashemi,A.R.M., 2005. Geometric Correction in IKONOS Images-Case Study:Tehran, Iran. In: FIG working week 2005 and GSDI-8, April 16-21 April 2005, Cairo, Egypt .

Hongyan Wen, 2009.Research on Mosaic Algorithm of Remote Sensing Image. Huazhong University of Science \& Technology, Wuhan.

Ping Wang, Yuhuan Li, Yongguo Zheng and Jixian Zhang, 2003. Intelligent information extraction of LUCC. Journal of Shandong Agricultural University (Natural Science Edition), Shandong, (3),pp.406-409.

Fang Jiang and Guoyong Zhang, 2002. Some Noteworthy Problems in Visual Interpretation of Remote Sensing Image. Journal of Changchun Institute of Technology (Natural Science Edition), Changchun, 3(3),pp.49-50. 